\title{
Cavity Effect on Phase Noise of Fabry-Perot Modulator-based Optical Frequency Comb
}

\author{
Joonyoung Kim, David J. Richardson, and Radan Slavík \\ Optoelectronics Research Centre, University of Southampton, Southampton, United Kingdom \\ j.kim@soton.ac.uk
}

\begin{abstract}
We study previously unconsidered filtering effect of a Fabry-Perot (FP) cavity on the phase noise of optical frequency comb generated with an FP-based electro-optic modulator. We found that phase noise can be suppressed by up to $30 \mathrm{~dB}$ for offset frequencies $>\mathrm{FSR} /$ finesse.
\end{abstract}

Keywords-Optical frequency comb; Fabry-Perot electro-optic modulator; phase noise.

\section{INTRODUCTION}

For more than two decades, an electro-optic phase modulator enclosed in a short Fabry-Perot cavity (FP-EOM) has proven a valuable tool for numerous applications [1]. This is mainly because it can efficiently generate an optical frequency comb that covers the entire $C$ band $(1530 \mathrm{~nm}-1565$ $\mathrm{nm}$ ) using a relatively low power microwave (RF) drive signal $(<1 \mathrm{~W})$. The generated comb has a well-defined comb tone spacing and output power in excess of $-20 \mathrm{dBm}$, allowing each tone to be used for onward processing.

The generated comb phase noise properties in the temporal domain have been studied in detail [2]. The jitter originating in the FP-EOM was found to be extremely low ( $6 \mathrm{fs}$, integrated from $1 \mathrm{~Hz}$ to $10 \mathrm{MHz}$ ) [2]. As for the frequency (Fourier) domain, which gives information regarding the phase noise properties of individual comb tones, there are two main contributions to the phase noise. The first comes from the phase noise of the seed light which is 'copied' onto each comb tone [3]. Thus, the use of a seed light source with well stabilized frequency/phase is needed for low noise operation of the FP-EOM. The other noise source is noise originating in the RF driving signal. The phase noise of the RF drive signal may be low, but it is amplified by $20 \log [k]$, where $k$ is the comb tone number with respect to the seed signal [4]. Thus, higherorder comb tones normally suffer from significantly higher phase noise as compared to the low-order comb tones.

In this paper, we show by numerical simulations that this picture does not actually describe the phase noise properties of the generated optical comb fully, as it neglects the optical filtering characteristics of the FP cavity itself. This is to the best of our knowledge the first time this phenomenon that leads to beneficial lowering of phase noise is investigated.

\section{THEORY}

First, we modify the existing equations describing FP-EOM that will allow us to insert the phase variations (noise) of the input RF signal.
The output electric field of the FP-EOM after $N$ round-trips can be written as:

$$
E_{\text {out }}=\left.E_{t}\right|_{\tau=N \tau_{r}} \sqrt{(1-R) \eta} \exp \left\{-j\left(\pi \Delta f_{o} / F S R+\phi_{N+1}\right)\right\}
$$

where, $R$ is the reflectivity of the mirror, $\eta$ is the single-pass power transmission efficiency in the waveguide, $\Delta f_{o}$ is the frequency detuning between the seed light and the FP cavity, FSR is the free spectral range of the FP cavity, $\phi$ is the phase modulation, and $\tau_{r}$ is the round-trip time. The electric field inside the cavity after $N$ round-trips, $\left.E_{t}\right|_{\tau=N \tau_{r}}$, can be presented as:

$$
\begin{aligned}
& \left.E_{t}\right|_{\tau=N \tau_{r}}=E_{\text {in }} \sqrt{1-R} \\
& \quad+\left.R \eta E_{t}\right|_{\tau=(N-1) \tau_{r}} \exp \left\{-j\left(2 \pi \Delta f_{o} / F S R+\phi_{N}\right)\right\}
\end{aligned}
$$

where $\left.E_{t}\right|_{\tau=(N-1) \tau_{r}}$ is the electric field inside cavity at the previous round-trip. The phase modulation, $\phi$ is given by [3]:

$$
\phi_{q}=\beta \sin \left\{\begin{array}{l}
2 \pi f_{m} t-\frac{\pi f_{m}}{F S R}(2 N-2 q+3) \\
+\varphi(t-[2 N-2 q+3] / F S R / 2)
\end{array}\right\}
$$

where $\beta$ is the modulation index, $f_{m}$ is the modulation frequency and $\varphi$ the phase of the RF drive signal. The phase noise of the RF drive signal was added via $\varphi$ as white Gaussian noise (WGN) generated over the bandwidth of interest (e.g. >2 $\times$ FSR). In this way, phase shift/modulation and loss imposed by the resonator are updated each round trip and added to the input field (into the FP cavity). Note that the phase modulation was presented without considering the backward-directional travelling wave with the assumption that $f_{m} \approx$ integer multiple of FSR of the cavity. Here, the phase noise of the seed light was not considered.

\section{RESUlTS}

In our simulations, the modulation frequency $\left(f_{m}\right)$ was set to 10 times the FSR (which corresponds to devices available commercially, e.g., $2.5 \mathrm{GHz}$ FSR and $25 \mathrm{GHz}$ tone spacing). The reflectivity of the mirrors $(R)$ and the transmission power efficiency of the waveguide $(\eta)$ were about $97 \%$, and $97.6 \%$, respectively, where the corresponding finesse was about 56, which are again typical parameters for commercially-available devices. 

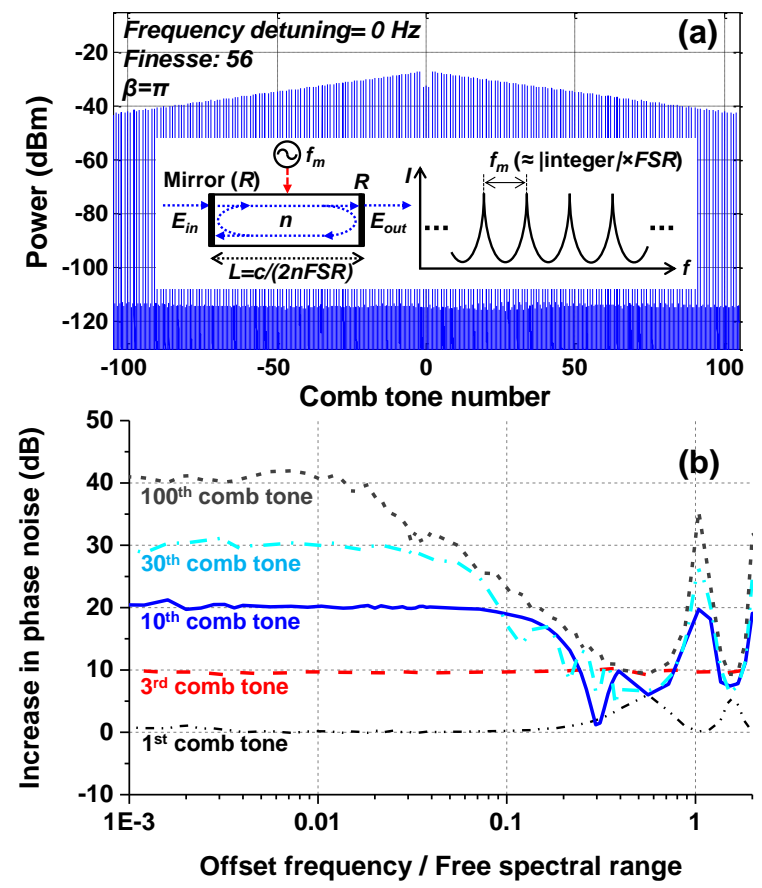

Fig. 1. Simulation results: (a) output spectrum and (b) calculated phase noise increment at the various comb tones.

When the frequency detuning $\left(\Delta f_{o}\right)$ is $0 \mathrm{~Hz}$, given that the modulation index $(\beta)$ is set to be $\pi$, the widest spectrum (i.e. shortest pulses) is obtained, Fig. 1(a). The incident power to the FP-EOM was set to $20 \mathrm{~mW}(13 \mathrm{dBm})$, and the total output power was about $-13 \mathrm{dBm}$. The inset in Fig. 1(a) is the simplified diagram on the operation of FP-EOM. After reaching the steady-state, we analyzed each comb tone in the frequency domain. We compared the phase noise of each comb tone with the phase noise of the RF drive signal.

As in Fig. 1(b), the phase noise at low offset frequency (i.e. $<\mathrm{FSR} /$ finesse) is increased by $20 \log [k]$ as the comb tone number increases by $k$, in line with previously-published expectations. However, from about the $10^{\text {th }}$ comb tone, the phase noise does not increase uniformly across the whole frequency range. Instead, the phase noise at high offset frequency (>FSR/finesse) is suppressed due to the FP resonator filtering characteristics, which has not been suggested/analyzed/observed before. This feature is more significant for higher-order comb tones. For an example, the added phase noise of the $100^{\text {th }}$ comb tone is suppressed at offset frequencies higher than FSR/finesse, e.g. suppression of $30 \mathrm{~dB}$ occurs at $0.5 \times \mathrm{FSR}$. Even better noise suppression (as compared to that shown in Fig. 1(a)) is expected from higher finesse FP cavities.

We also calculated the phase noise increment at the $10^{\text {th }}$ comb tone for various values of frequency detuning $\left(\Delta f_{o}\right)$ and modulation index $(\beta)$, as in Fig. 2. With the frequency detuning increasing, Fig. 2(a), while other parameters are kept fixed, the phase noise suppression gets even better than for the zero detuning, and becomes the best for $\Delta f_{o}=(\beta / 2 \pi) \times \mathrm{FSR}$, that is the detuning at which the FP-EOM has the maximum
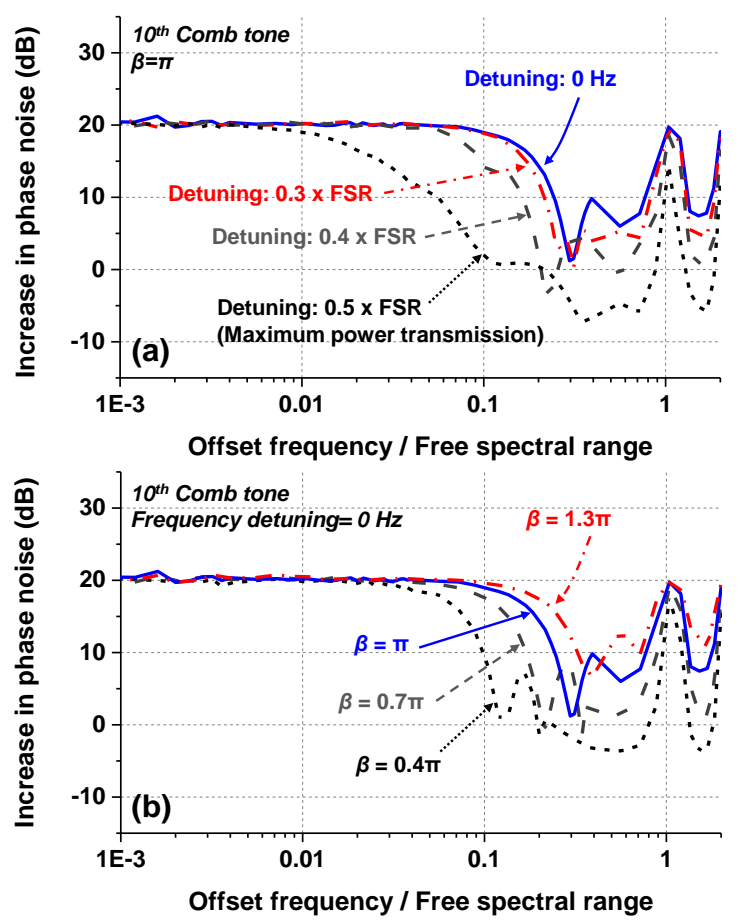

Fig. 2. Calculated phase noise increment at the $10^{\text {th }}$ comb tone (a) for various frequency detuning $\left(\Delta f_{o}\right)$ between the Fabry-Pérot cavity and seed light and (b) for various modulation index $(\beta)$.

transmission. As shown in Fig. 2(b), the phase noise suppression improves also with modulation index reduction. Although these conditions (i.e. maximum FP-EOM transmission frequency and small modulation index) produce a narrower comb, they may be of interest if a smaller number of comb tones and low phase noise are required.

\section{CONCLUSION}

We studied the cavity effect on the phase noise for an optical frequency comb based on the Fabry-Perot electro-optic modulator (FP-EOM). At low offset frequency, the phase noise increased by $20 \log [k]$, as the comb tone number increases by $k$. However, at high offset frequency (> FSR/finesse), the phase noise is reduced due to the FP-EOM cavity filtering by up-to $30 \mathrm{~dB}$ at the $100^{\text {th }}$ comb tone. In our further work we plan to carry out experimental confirmation of our simulations and expect these to be available at the time of the conference.

\section{REFERENCES}

[1] J. Ye, H. Schnatz, and L. W. Hollberg, "Optical Frequency Combs: From Frequency Metrology to Optical Phase Control," IEEE J. Sel. Top. Quantum Electron. 9, 1041 (2003).

[2] S. Xiao, L. Hollberg, N. R. Newbury and S. A. Diddams, "Toward a low-jitter $10 \mathrm{GHz}$ pulsed source with an optical frequency comb generator," Opt. Express 16, 8498 (2008).

[3] T. Saitoh, S. Mattory, S. Kinugawa, K. Miyagi, A. Taniguchi, M. Kourogi, and M. Ohtsu, "Modulation Characteristic of Waveguide-Type Optical Frequency Comb Generator,” J. Lightwave Technol. 16, 824 (1998).

[4] J. Kim, G. Marra, D. S. Wu, D. J. Richardson, and R. Slavik, "Wavelength conversion technique for optical frequency dissemination applications," Opt. Lett. 41, 1716 (2016). 
